\title{
An Alternative Approach to Understanding the Observed Positron Fraction
}

\author{
Martin H. Israel
}

Received: 28 April 2014 / Published online: 25 June 2014

(C) Sociedade Brasileira de Física 2014

\begin{abstract}
Space-based observations by PAMELA (Adriani et al., Nature 458, 607, 2009), Fermi-LAT (Ackerman et al., Phys. Rev. Lett. 105, 01103, 2012), and AMS (Aguilar et al., Phys. Rev. Lett. 110, 141102, 2013) have demonstrated that the positron fraction (e+/total-e) increases with increasing energy above about $10 \mathrm{GeV}$. According to the propagation model for Galactic cosmic rays in widespread use (Moskalenko \& Strong, Astrophys. J. 493, 693, 1998), the production of secondary positrons from interaction of cosmic-ray protons and heavier nuclei with the interstellar medium gives a generally falling positron fraction between 10 and $100 \mathrm{GeV}$, with secondary positrons accounting for only $\sim 20 \%$ of the observed positron fraction at $100 \mathrm{GeV}$; so some other physical phenomena have been proposed to explain the data. An alternative approach to interpreting the positron observations is to consider these data as presenting an opportunity for re-examining models of Galactic cosmicray propagation. Following release of the PAMELA data, three groups published propagation models (Shaviv, et al., Phys. Rev. Lett. 103, 111302, 2009, Cowsik and Burch, Phys. Rev. D. 82, 023009, 2010, Katz et al., Mon. Not. R. Aston. Soc. 405, 1458 2010) in which the observed positron fraction is explained entirely by secondary positrons produced in the interstellar medium. In May of this year, stimulated by the AMS extension of the positron data to higher energy with excellent statistics, two of those groups presented further development of their calculations (Cowsik et al. 2013, Blum et al. 2013), again concluding that the observed positrons can be understood as secondaries. None of the authors of these five papers was registered for the 33rd
\end{abstract}

M. H. Israel (凶)

Department of Physics and the McDonnell Center for the Space Sciences, Washington University in St. Louis, St. Louis, MO USA e-mail: mhi@physics.wustl.edu
International Cosmic Ray Conference (ICRC). Although I am not an author of any of these papers, I have some close familiarity with one of these recent papers, so the conference organizers invited me to bring this alternative approach to the attention of the conference. The present paper is a summary of the material I presented, along with a brief comment about reaction at the conference to this approach.

Keywords Positron fraction · Cosmic-ray positrons . Galactic cosmic-ray propagation

\section{Introduction}

Recent measurement of cosmic-ray positrons by the AMS-02 magnetic spectrometer on the International Space Station [3] confirms, with excellent precision, earlier measurements by PAMELA [1] and by Fermi-LAT [2] that demonstrate a positron fraction, $\mathrm{e}+/(\mathrm{e}++\mathrm{e}-)$, increasing with energy from 0.05 at $10 \mathrm{GeV}$ to 0.15 between 200 and $350 \mathrm{GeV}$. Some cosmic-ray positrons must be created in interstellar space as secondary products of interaction of cosmic-ray nuclei (mostly protons and helium nuclei) with nuclei of the interstellar medium (mostly hydrogen and helium). However, widely used models of propagation of cosmic rays in the Galaxy, for example [4], predict that such secondary positrons would give a positron fraction falling in this energy range from about 0.04 to less than 0.03 . Thus, other explanations for the rising positron fraction must be considered. This "highlight talk" was invited to the ICRC for the purpose of bringing attention to recent papers (none of which were written by the presenter of this "highlight talk") that explain the rising positron fraction by modifying the widely used Galactic propagation model. Before presenting those alternative propagation models, we first 
summarize very briefly the other explanations for the rising positron fraction that have been discussed in the literature and in this ICRC.

\section{Primary Positrons}

The most widely discussed interpretation of the rising positron fraction is that there could be a new primary source of positrons, in addition to the secondary positrons produced by nuclear interactions. Two such primary sources have been considered annihilation or decay of dark matter particles, and positrons produced in pulsar magnetospheres.

Dark-matter origin of the positron excess has been widely discussed (for example $[10,11]$ ). The definitive evidence for a dark-matter origin would be an abrupt drop in the positron fraction above some energy, since such positrons cannot have energy greater than the rest mass of the darkmatter particles. No evidence for such a drop has been seen in AMS data up to about $300 \mathrm{GeV}$, but with time AMS could extend their measurements to about $1 \mathrm{TeV}$ so a dark-matter signature could yet be found.

In pulsar magnetospheres, electrons can be accelerated and emit energetic photons by curvature radiation in the pulsars strong magnetic field. The resulting electromagnetic cascade would produce positrons, and acceleration of the positrons thus produced can yield a hard spectrum that could account for the rising positron fraction. See, for example [12-14].

\section{Secondary Positrons Produced in the Sources}

An alternative interpretation of the rising positron fraction is the production of secondary positrons in the cosmicray source regions $[15,16]$. In addition to the secondary positrons produced by cosmic rays interacting in interstellar space, there are likely to be secondary positrons produced by interactions with material in the source regions. These secondary positrons would then participate in the same acceleration process that produces cosmic-ray protons and electrons. The positrons thus accelerated are likely to have a harder spectrum than the Galactic secondaries calculated by Moskalenko and Strong and could thus account for rising positron fraction.

Erlykin and Wolfendale (private communication, hallway conversation at the ICRC) have submitted a paper proposing another mechanism for secondary positrons produced at the cosmic-ray sources. They point out that interactions of primary cosmic-ray nuclei in the source region will produce short-lived secondary nuclei, some of which will decay by positron emission. The $\mathrm{MeV}$ positrons thus produced will be very efficiently accelerated to cosmic-ray energies.

\section{Secondary Positrons Produced in the Interstellar Medium}

Models of cosmic-ray propagation in the Galaxy have been developed over the years to account for the observed secondary particles that result from cosmic-ray primaries interacting with the medium through which they propagate nuclei like boron, antiprotons, gamma rays, etc. Since at least some of the cosmic-ray positrons must be such secondary particles, it seems reasonable to investigate whether the recent positron observations can be accommodated within a Galactic propagation model. In other words, it seems appropriate to treat these observed positrons as one more constraint on cosmic-ray propagation.

Three approaches, each different from the others, have been proposed that account for the observed rise in positron fraction above $10 \mathrm{GeV}$ as being due entirely to secondary positrons produced by interactions of primary cosmic rays throughout the Galaxy. It must be noted here that during the question period following the oral presentation of this "highlight paper" at the ICRC, objections to each of these three approaches were raised. These objections have been summarized in one section of a subsequent paper [17]. While I have received counterarguments to some of those objections from at least one of the authors, I will leave it to the authors to publish their own defenses as they consider appropriate.

One approach ignores the details of propagation models and simply assumes that positrons are produced at the same places as secondary nuclei like boron (which is the fragmentation product of carbon, oxygen, and other heavier nuclei interacting with interstellar gas). From the ratio of production of positrons to production of boron, one infers an upper limit to the positron fraction. It is an upper limit because it ignores energy loss processes suffered by positrons but not by nuclei. The upper limit thus calculated matches the peak of the positron fraction observed by AMS-02 [7, 9]. In support of this model, the authors apply the same principle to calculating the flux of cosmic-ray antiprotons, and produce a calculated antiproton/proton spectrum in excellent agreement with the result from PAMELA [18]. They point out that while the primary positron models, whether from dark matter or from pulsar magnetospheres, make no quantitative prediction of the positron fraction, this simple propagation approach gives excellent quantitative agreement with the peak positron ratio observed. 
While the positron fraction thus calculated, ignoring energy losses, agrees closely with the observation at around 200 to $300 \mathrm{GeV}$, it falls substantially below the observation at lower energy, by about a factor of four at $10 \mathrm{GeV}$. Taking account of positron energy loss by synchrotron radiation and inverse Compton scattering, this model thus implies an escape time from the Galaxy of $>\sim 1 \mathrm{Myr}$ at $\sim 200 \mathrm{GeV}$, increasing with decreasing energy, reaching $<\sim 30 \mathrm{Myr}$ at $10 \mathrm{GeV}$.

A second approach notes that the Moskalenko \& Strong model of Galactic cosmic-ray propagation is cylindrically symmetrical, but in fact cosmic-ray sources are likely to be concentrated in the spiral arms of the Galaxy. Taking into account energy loss of primary cosmic-ray electrons from those sources, while noting that secondary positrons are produced from primary nuclei that are distributed more uniformly throughout the Galaxy, a model has been developed that produces a rising positron fraction above $10 \mathrm{GeV}$ [5]. It must be noted, however, that another calculation of Galactic cosmic-ray propagation, in which the spiral structure is incorporated, fails to match the observed positron fraction without invoking some primary positron source [19]. Other similar calculations that failed to match the rising positron fraction were presented at this ICRC $[20,21]$.

A third modification of the commonly used Galactic propagation model invokes the "Nested Leaky Box" model [22]. In this model, boron and other nuclear secondaries are primarily produced in "cocoons" around the sources, from which escape is energy dependent, going as $\sim \mathrm{E}^{-0.6}$. This energy-dependent escape from the secondaryproduction region accounts for the observed energy dependence of the secondary/primary cosmic-ray nucleon ratio (e.g., boron/carbon) at the energies where this ratio is observed, up to a few hundred $\mathrm{GeV} /$ nucleon. In this model, only a small fraction of the secondary nuclei are produced in the interstellar medium of the Galaxy, from which escape is essentially independent of energy.

These authors $[6,8,24]$ note that while nuclear interactions produce secondary nuclei at essentially the same energynucleon as the primary interacting nucleus, secondary positrons are generally produced with about $5 \%$ of the energy of the primary nuclei (usually protons) whose interaction produced them. Thus positrons observed with energies between 10 and $300 \mathrm{GeV}$ are the product of interacting nuclei of energy between roughly 200 and $6,000 \mathrm{GeV}$. In this Nested Leaky Box model, nuclei of those very high energies quickly escape the "cocoons" around the sources, so the positrons observed in the energy region where their fraction is rising have been produced by interactions in the interstellar region with density about 0.5 hydrogen atoms $/ \mathrm{cm}^{3}$, from which escape time is about $2 \mathrm{Myr}$ essentially energy independent. In this model, these authors easily reproduce both the observed $\mathrm{B} / \mathrm{C}$ ratio, and the positron fraction over the entire energy range for which we have observations, from 1 to $\sim 300 \mathrm{GeV}$.

Cowsik et al. point out that this Nested Leaky Box Model, with its energy-independent leakage from the Galaxy, avoids the inconsistency between the observed limits on cosmic-ray anisotropy at energies above a $\mathrm{TeV}$ and the higher values predicted in the more generally used propagation model (see for example, figure 12 in [23]).

\section{Conclusion}

At the same time that we consider positrons from possible new primary sources, such as dark matter or pulsar magnetospheres, we should also be using the new positron data to reexamine cosmic-ray propagation models. In other words, it would be desirable to see whether with detailed calculations a model can be developed that is consistent with the observed positrons as secondaries, along with other secondaries antiprotons, deuterium, boron, and other nuclear secondaries and gamma rays. We have seen three different approaches for modifying the widely used propagation model that do indeed treat the positrons as secondary. None of these three approaches is yet a fully developed model. All three of them, as well as the widely used model of Moskalenko \& Strong, should be studied further before we can be certain that the positrons come from some new source.

Acknowledgments I greatly appreciate the invitation to present this paper; finding myself in the position of spokesman for the work of others led to many enlightening conversations at the conference as well as before and after it. I thank Ramanath Cowsik, Kfir Blum, and Tsvi Piran for helpful discussions of their work. I am grateful to Igor Moskalenko and Pasquale Blasi for informative exchanges of ideas. Preparation of this"highlight paper" was supported in part by several NASA grants, and in part by the McDonnell Center for the Space Sciences at Washington University.

\section{References}

1. O. Adriani et al. Nature. 458, 607 (2009)

2. M. Ackerman et al. Phys. Rev. Lett. 105, 01103 (2012)

3. M. Aguilar et al. Phys. Rev. Lett. 110, 141102 (2013)

4. I. V. Moskalenko, A. W. Strong. Astrophys. J. 493, 693 (1998)

5. N. J. Shaviv, E. Nakar, T. Piran. Phys. Rev. Lett. 103, 111302 (2009)

6. R. Cowsik, B. Burch. Phys. Rev. D. 82, 023009 (2010). arXiv:0908.3494

7. B. Katz, K. Blum, J. Morag, E. Waxman. Mon. Not. R. Aston. Soc. 405, 1458 (2010) 
8. R. Cowsik, B. Burch, T. Madziwa-Nussinov. arXiv:1305.1242 (2013)

9. K. Blum, B. Katz, E. Waxman. arXiv:1305.1324 (2013)

10. L. Bergstrom, J. Edsjo, G. Zaharijas. Phys. Rev. Lett. 103, 031103 (2009)

11. A. Hektor et al. (2013). arXiv:1307.2561

12. D. Hooper, P. Blasi, P. D. Serpico. JCAP. 01, 025 (2009)

13. I. Cholis, D. Hooper (2013). arXiv: 1304.1840

14. Q. Yuan et al. (2013). arXiv: 1304.1482

15. P. Blasi. Phys. Rev. Lett. 103, 051104 (2009)

16. Berezhko \&amp; Ksenofontov, paper 815 of this ICRC
17. I. V. Moskalenko. arXiv: 1308.5482

18. Adriani et al. JETP Lett. 96, 621 (2013)

19. D. Gaggero et al. Phys. Rev. Lett. 111, 021102 (2013)

20. D. Grasso, paper 559 of this ICRC

21. Gebauer, Kunz \&amp; Weinreuter, paper 1115 if this ICRC

22. R. Cowsik, L. W. Wilson, Proc. 13th Int. C. R. Conf. 174 \& 1500 (1973)

23. A. W. Strong, I. V. Moskalenko, V. S. Ptuskin. Annu. Rev. Nucl. Part. Sci. 57, 285 (2007)

24. R. Cowsik, B. Burch, T. Madziwa-Nussinov. Astrophys. J. 786, 124 (2014) 